\title{
Ultra-short Wavelength Operation of Thulium-doped Fibre Amplifier in the 1628-1655nm Waveband
}

\author{
S. Chen ${ }^{(1,2 *)}$, Y. Jung( ${ }^{(1)}$, S.U.Alam ${ }^{(1)}$, R.Sidharthan(2), D. Ho(2), J.M.O.Daniel(3), S. Yoo(2), and D. J. \\ Richardson ${ }^{(1)}$
}

(1) Optoelectronics Research Centre, University of Southampton, SO171BJ, United Kingdom

(2) The Photonics Institute, School of Electrical and Electronic Engineering, Nanyang Technological University, Singapore 639798

(3) Jay's affilation

\section{*sc4f15@soton.ac.uk}

\begin{abstract}
We present a silica-based thulium-doped fibre amplifier in the 1628-1655nm waveband based on $\mathrm{Tm} / \mathrm{Ge}$ co-doped fibre. Up to $19 d B$ external small signal gain and a noise figure of $4.4 d B$ are achieved at $1655 \mathrm{~nm}$.
\end{abstract}

\section{Introduction}

With the growing demand for capacity in optical fibre transmission, considerable research has been devoted to the 1.6-2 $\mu \mathrm{m}$ wavelength region as an emerging transmission window for next generation optical communications based on hollow-core photonic-bandgap fibres (HCPBGFs) ${ }^{1}$. As the low loss window of silica fibre extends up to $1.7 \mu \mathrm{m}$, and with the continual improvement of background loss in HC-PBGFs, the possibility of future optical communications networks operating seamlessly from 1.55 to $2 \mu \mathrm{m}$ is emerging, requiring efficient optical fibre amplifiers covering the entire $1.55-2 \mu \mathrm{m}$ waveband.

To date, the L-band erbium doped fibre amplifier (EDFA) can only covers wavelength up to $1.62 \mu \mathrm{m}$, and various configuration of thuliumdoped fibre amplifiers (TDFAs) have been demonstrated offering high gain and low noise performance across the wavelength 1700-2050 $\mathrm{nm}$ range ${ }^{2,3}$. With effective management of amplified spontaneous emission (ASE), the short-wavelength amplification edge of the TDFA has further extended down to $1650 \mathrm{~nm}$ with a small signal gain of $8 \mathrm{~dB}$ demonstrated $\mathrm{d}^{4}$. This is a great improvement but a $\sim 30 \mathrm{~nm}$ spectral gap still exists between operating windows of the EDFA and TDFA.. Very recently, we have explored a new glass composition (particularly, a thulium $(\mathrm{Tm})$ and germanium (Ge) co-doped silica fibre) and successfully demonstrated a tunable Tmdoped fibre laser in the wavelength range of 1620-1660 $\mathrm{nm}^{5}$. With the distinctive feature of blue-shifted absorption and emission spectra, the $\mathrm{Tm} / \mathrm{Ge}$ co-doped fibre provides the intriguing possibility of bridging the gap between the long wavelength edge of the L-band EDFA and short wavelength edge of the TFDA.

In this paper, we experimentally demonstrate ultra-short wavelength operation of a TDFA and present the first realization of a silica-based TDFA operating down to as short as $1628 \mathrm{~nm}$.

\section{Fibre characterisation}

The $\mathrm{Tm} / \mathrm{Ge}$-doped fibre was fabricated in-house using the conventional MCVD technique in conjuction with solution doping (dopant

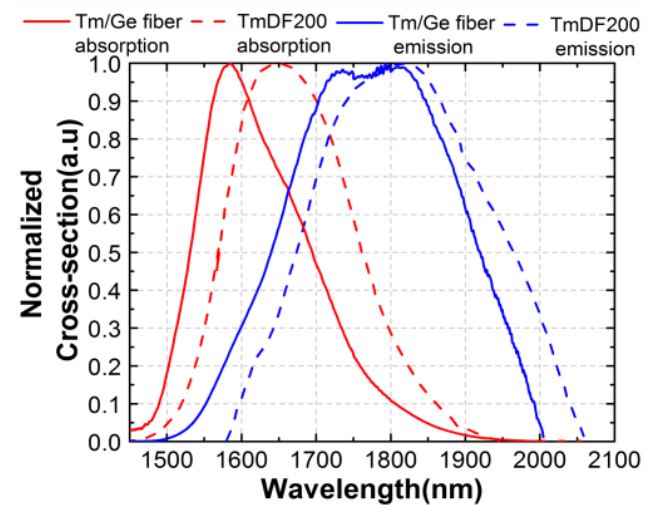

(a)

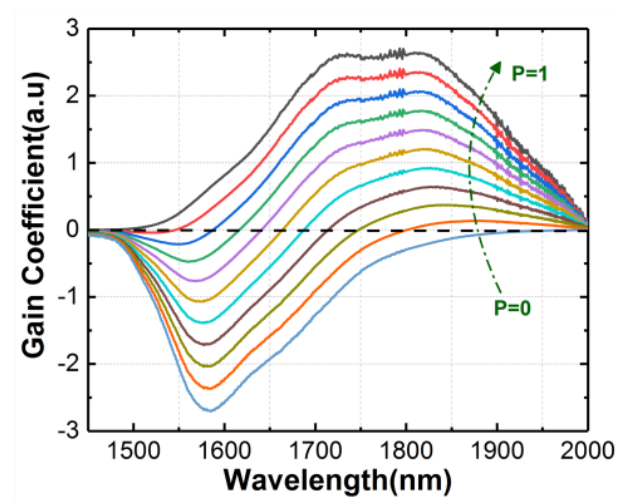

(b)

Fig. 1(a) normalized absorption and emission cross-sections for Tm-doped fiber (OFS TmDF200) and in-house fabricated Tm/Ge co-doped fiber, respectively, and (b) the calculated normalized gain coefficient of Tm/Ge codoped fiber. 
concentration of $\sim 0.004 \mathrm{~mol} \%$ for $\mathrm{Tm}_{2} \mathrm{O}_{3}$ and $\sim 19$ $\mathrm{mol} \%$ for $\mathrm{GeO}_{2}$ ). The fibre has a core diameter of $4.4 \mu \mathrm{m}$ and an NA of $\sim 0.28$. As compared to commercial Tm-doped aluminosilicate fibre (OFS TmDF200), the absorption spectrum of the $\mathrm{Tm} / \mathrm{Ge}$ co-doped fibre is significantly blue shifted (70 nm shorter) as shown by the red solid line in Fig. 1(a) with a peak absorption of $\sim 20 \mathrm{~dB} / \mathrm{m}$ at $1580 \mathrm{~nm}$. According to the McCumber theory, the derived emission cross-section of $\mathrm{Tm} / \mathrm{Ge}$ codoped fibre is also blue-shifted by about $50 \mathrm{~nm}$ compared to TmDF200 fibre (blue solid line in Fig. 1(a)). Therefore, it is anticipated that the proposed $\mathrm{Tm} / \mathrm{Ge}$ co-doped fibre can be used for efficient signal amplification at wavelengths much shorter than $1650 \mathrm{~nm}$.

Firstly, it is useful to compute the wavelength dependence of net gain as a function of population of the upper laser level (populaiton inversion). The net gain coefficient, $G(\lambda)$, can be expressed by the following equation 6 :

$$
\mathrm{G}(\lambda)=\mathrm{N}\left[\mathrm{p} \sigma_{\mathrm{e}}(\lambda)-(1-\mathrm{p}) \sigma_{\mathrm{e}}(\lambda)\right]
$$

where $p$ represents the population of the upper laser level, and $\mathrm{N}$ stands for the total number of $\mathrm{Tm}^{3+}$ ions. As shown in Fig. 1(b), the gain spectrum strongly depends on the population inversion and the gain peak shifts to shorter wavlenghts as the inversion level is increased. Therefore to achieve efficient signal amplification below $1700 \mathrm{~nm}$, more than $30 \%$ population inversion is required necessitating optimization of the fibre length for a given pump wavelength.

\section{Experiment setup}

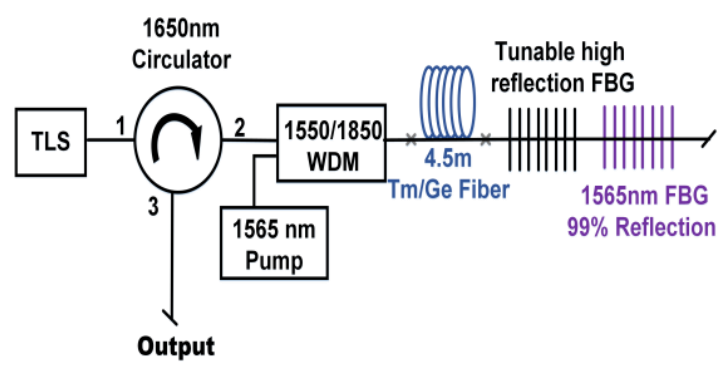

(a)

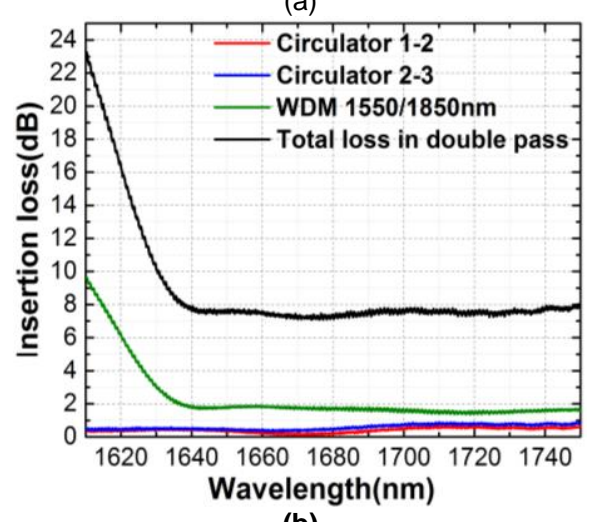

(b)
Fig. 2 (a) Schematic of the TDFA, TLS: tunable laser source; (b) insertion losses of the WDM coupler (green) and circulator (blue and pink) used in the setup, and the total optical loss of the double pass cavity.

Fig. 2(a) shows a schematic of the TDFA. In our experiment, a commercially available tunable laser source (Tunics T100S-HP) covering the wavelength range of $1500-1680 \mathrm{~nm}$ was used as a seed source with an input power of $-20 \mathrm{dBm}$ and $0 \mathrm{dBm}$ for small and saturated signals, respectively. An optical circulator optimized at $1650 \mathrm{~nm}$ and with high optical isolation ( $>40 \mathrm{~dB}$ at center wavelength) and low insertion loss $(0.45$ $\mathrm{dB}$ ) was employed as depicted in Fig. 2(b). In order to extract enough gain at short wavelengths, a high population inversion is required and an in-house built high-power $\mathrm{Er} / \mathrm{Yb}$ fibre laser operating at $1565 \mathrm{~nm}$ was used as a pump source with a maximum output power of 4.35 W (36.3 dBm). A dielectric filter-based $1550 / 1850 \mathrm{~nm}$ wavelength division multiplexer (WDM) was used for combining the pump and signal. As shown in Fig. 2(b), the insertion loss of the WDM coupler was less than $2 \mathrm{~dB}$ but this gradually increased at wavelengths shorter than $1640 \mathrm{~nm}$. In addition, Fig. 2(b) also shows the total optical loss in a double pass configuration including the loss of an optical circulator, a $1550 / 1850 \mathrm{~nm}$ WDM coupler as well as the optimized splicing loss of $0.8 \mathrm{~dB}$ between standard SMF and Tm/Ge co-doped fibres.

To realize short wavelength operation, a tunable fibre Bragg grating (FBG) was used in our experiment as an ASE filter as well as a narrowband signal retroreflector to realize a double pass implementation.

The FBG can be tuned from $1620 \mathrm{~nm}$ through to $1660 \mathrm{~nm}$ by using an axial compression mechanism. The measured reflectivity and bandwidth of the FBG varied from $91 \%$ to $94 \%$ and from $0.67 \mathrm{~nm}$ to $1 \mathrm{~nm}$ respectively, depending on the operating wavelengths. Moreover, a $1565 \mathrm{~nm}$ FBG with 99\% reflection was incorporated to recycle the residual pump light $(\sim 0.4 \mathrm{~W})$. The external gain and NF of the amplifier was measured by an optical spectrum analyzer (Yokogawa AQ6375) and a thermal power meter (Ophir 3A-FS).

\section{Result and discussion}

Figure 3 shows the gain and noise figure (NF) performance of the proposed TDFA. An external small-signal gain of $19 \mathrm{~dB}$ was achieved at $1655 \mathrm{~nm}, 8 \mathrm{~dB}$ at $1632 \mathrm{~nm}$ and $4 \mathrm{~dB}$ at $1628 \mathrm{~nm}$. Compared to our previous demonstration of a short-band TDFA ${ }^{3}$, we have successfully extended the short wavelength edge of the silicabased TDFA from $1650 \mathrm{~nm}$ to $1628 \mathrm{~nm}$. The 


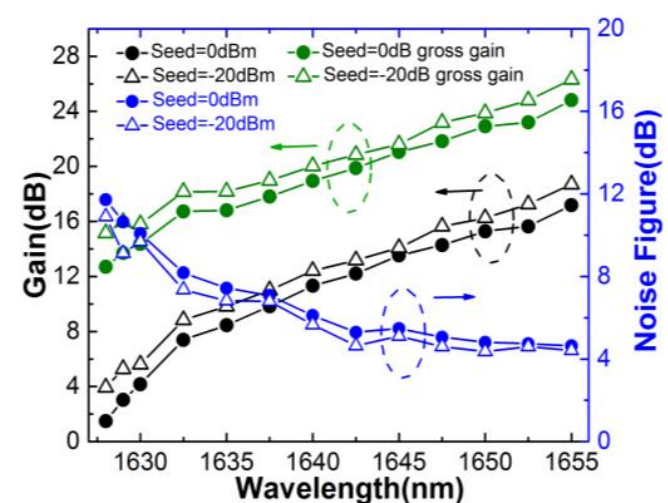

(a)

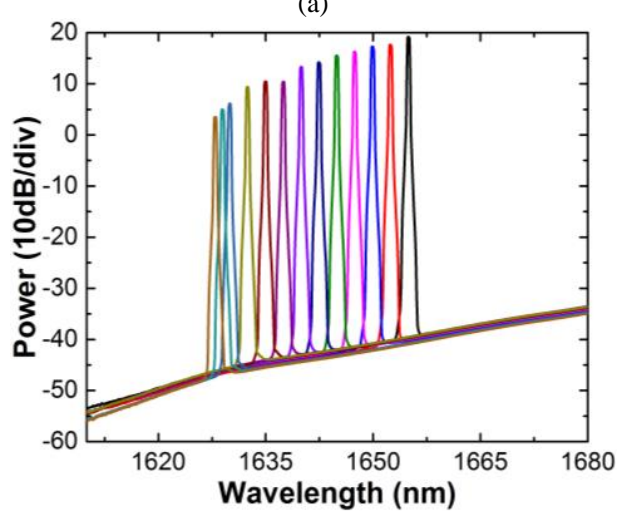

(b)

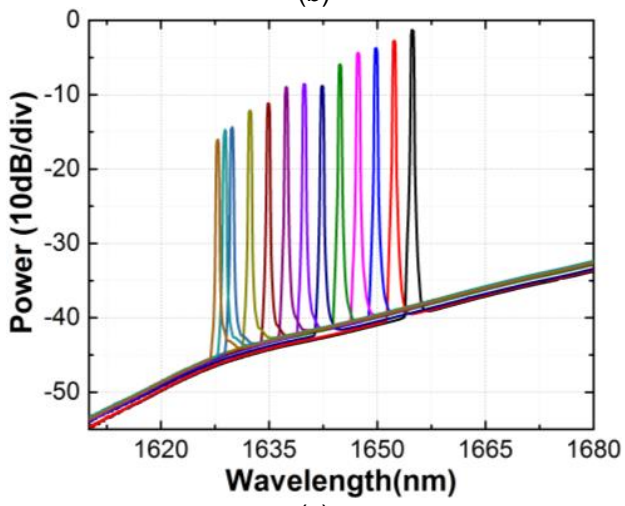

(c)

Fig. 3 (a) Gain and NF performances of the TDFA Amplified spectra for (b) saturated and (c) small signals, measured with $0.5 \mathrm{~nm}$ OSA resolution.

saturated gain shows similar behavior and varies from $7 \mathrm{~dB}$ to $17.2 \mathrm{~dB}$ with only $2.5 \mathrm{~dB}$ difference as compared to the small signal gain. The external NFs for saturated signal and small signal were as low as $4.6 \mathrm{~dB}$ and $4.4 \mathrm{~dB}$ at a wavelength of $1655 \mathrm{~nm}$ but increased towards shorter wavelengths. This is mainly due to the high insertion loss of the WDM coupler and strong reabsorption of signal light by Tm ions in their ground state. By considering the total insertion loss of our amplifier with the double pass architecture, the gross (or internal) small-signal gain is around $15-26 \mathrm{~dB}$ in the $1628-1665 \mathrm{~nm}$. Therefore, further improvement in the performance of the TDFA is expected with higher pump powers (or indeed a different choice of pump wavelength such as $793 \mathrm{~nm}$, which has been theoretically investigated ${ }^{7}$ with the conclusion that the gains at shorter wavelengths will be enhanced compared to $1550 \mathrm{~nm}$ pumping), further optimization of both the passive fibre components and splicing loss between the active and passive fibres. Figures $3(\mathrm{~b})$ and (c) show the amplified optical spectra for the saturated and small signal regime, respectively. The amplified small signal has 30-38dB in-band optical signalto-noise ratio (OSNR) across the entire amplification band while the amplified saturated signal exhibits over $50 \mathrm{~dB}$ in-band OSNR.

\section{Conclusions}

With a new glass composition (i.e. Tm/Ge codoped fibre), we have successfully blue-shifted the emission cross-section of TDF compared to conventional (aluminosilicate based) Tm-doped fibre. By constructing a double-pass amplifier, we have demonstrated a silica-based TDFA operating from $1628 \mathrm{~nm}$ to $1655 \mathrm{~nm}$, a 2THz gain bandwidth extension compared to the previous best report. Up to $19 \mathrm{~dB}$ small signal gain (external) and a NF as low as $4.4 \mathrm{~dB}$ were achieved at $1655 \mathrm{~nm}$ with $>30 \mathrm{~dB}$ in-band OSNR.

\section{Acknowledgements}

This work is supported by the EPSRC funded AirGuide Photonics_(EP/P030181/1) Programme Grant and by the Academic Research Fund Tier 1, Ministry of Education (Singapore).

\section{References}

[1] F. Poletti, et al., "Towards high-capacity fibre-optic communications at the speed of light in vacuum," Nat.Photonics 7,279-284(2013).

[2] J. Wang, et al, "Broadband silica-based thulium doped fibre amplifier employing multi-wavelength pumping," Opt. Express 24, 23001 (2016).

[3] Y. Jung, et al, "Silica-Based Thulium Doped Fibre Amplifiers for Wavelengths beyond the L-band," in OFC (2016), M3D.5.

[4] Z. Li, et al, "Exploiting the short wavelength gain of silicabased thulium-doped fibre amplifiers," Opt. Lett. 41, 2197-2200 (2016).

[5] S. Chen, et al, "Ultra-short wavelength operation of a thulium doped fibre laser in the 1620-1660nm wavelength band," in OFC (2017), M2J.4

[6] Li, R. et al, "Mid-infrared emission properties and energy transfer evaluation in Tm3+ doped fluorophosphate glasses". J. Lumin. 162, 58-62 (2015).

[7] Yang et al, "Theoretical Characterization of the Ultra Broadband Gain Spectra at 1600-2100 nm from Thulium-Doped Fibre Amplifiers". IEEE Photonics Journal. PP. 1-1. 10.1109 (2016) 